\title{
Evaluation of New Monoclonal Antibody-Based Latex Agglutination Test for Detection of Cryptococcal Polysaccharide Antigen in Serum and Cerebrospinal Fluid
}

\author{
DEANNA L. KISKA, ${ }^{1}$ DEBORAH R. ORKISZEWSKI, ${ }^{1}$ DONNA HOWELL, ${ }^{1}$ AND PETER H. GILLIGAN ${ }^{1.2 *}$ \\ Clinical Microbiology and Immunology Laboratories, University of North Carolina Hospitals, ${ }^{\prime}$ and Departments of \\ Microbiology-Immunology and Pathology, University of North Carolina School of Medicine, ${ }^{2}$ Chapel Hill, \\ North Carolina 27514
}

Received 15 April 1994/Returned for modification 3 June 1994/Accepted 22 June 1994

\begin{abstract}
We evaluated the performance of CRYPTO-LEX (Trinity Laboratories, Inc., Raleigh, N.C.), a new mouse immunoglobulin $M$ monoclonal antibody latex agglutination reagent which reacts with the capsular polysaccharide of the four serogroups of Cryptococcus neoformans. This test was compared with CALAS (Meridian Diagnostics, Cincinnati, Ohio) for the ability to detect cryptococcal antigen in serum and cerebrospinal fluid (CSF). A total of 580 clinical specimens (327 serum and 253 CSF samples), primarily from human immunodeficiency virus-infected patients, were tested in this study. Sixty-seven specimens (44 serum and 23 CSF samples) were positive for cryptococcal antigen with both tests, and 511 (282 serum and 229 CSF samples) were negative. The two latex reagents agreed for 326 of 327 serum specimens (44 positives and 282 negatives). One serum specimen with a titer of 1:2 was CALAS positive but CRYPTO-LEX negative. The titer correlation coefficient for the two tests was 0.884 when two highly discordant serum specimens were eliminated from analysis of the data. The two latex tests agreed for 252 of 253 CSF specimens (23 positives and 229 negatives). One specimen with a titer of 1:2 was positive with CALAS and negative by CRYPTO-LEX. The correlation coefficient of the two tests for CSF titers was 0.886 . The sensitivity and specificity of CRYPTO-LEX were 97 and $100 \%$, respectively, with a $99.6 \%$ correlation with CALAS. These data show that the performance of CRYPTO-LEX is comparable to that of CALAS for detection of cryptococcal antigen in serum and CSF.
\end{abstract}

Cryptococcosis is a systemic infection caused by the encapsulated yeast Cryptococcus neoformans. The organism typically initiates infection by gaining entrance to the lungs, and from there, infection may rapidly disseminate to the brain and meninges. Prior to the AIDS epidemic, disseminated cryptococcal infection occurred only sporadically, typically in patients with sarcoidosis or lymphoma or in those receiving adrenal corticosteroids (10). However, there has been a dramatic increase in these cases since the 1980s, when AIDS became the leading predisposing factor to the development of this disease. Approximately 7 to $8 \%$ of human immunodeficiency virus (HIV)-infected patients develop cryptococcal meningoencephalitis, which often leads to death $(6,11,13)$. C. neoformans is now the pathogen most frequently isolated from cerebrospinal fluid (CSF) at our institution. Traditionally, the diagnosis of cryptococcosis has relied on culture of CSF or demonstration of encapsulated yeast cells in India ink preparations of CSF. Direct examination by India ink is a rapid but relatively insensitive method. It is positive in at least $50 \%$ of cases of cryptococcal meningitis in non-HIV-infected populations and 74 to $88 \%$ of cases in HIV-infected patients $(5-7,13,24)$. In addition, CSF chemistries and cell counts are often within normal parameters, precluding the use of these methods as rapid indicators of infection in HIV-infected individuals $(6,7$, 13). Detection of $C$. neoformans capsular polysaccharide antigen in CSF or serum has been established as a rapid and sensitive method for the diagnosis of cryptococcosis, exhibiting greater than $90 \%$ sensitivity $(6,12,13,23,24)$. Early methods for cryptococcal antigen detection included immunodiffusion,

\footnotetext{
* Corresponding author. Mailing address: Clinical Microbiology/ Immunology Laboratories, UNC Hospitals, 101 Manning Drive, Chapel Hill, NC 27514. Phone: (919) 966-5091. Fax: (919) 966-4526.
}

complement fixation, and latex agglutination $(2-4,16,17,19$, 21). Latex agglutination has gained widespread appeal because of its ease of use and greater sensitivity than the former methods. Commercially available cryptococcal antigen detection systems utilize either agglutination with anticryptococcal globulin-sensitized latex particles or enzyme immunoassay with polyclonal antibody capture and monoclonal detection $(14,15$, $18,20,22,23)$. Commercial kits typically demonstrate 90 to $100 \%$ sensitivity and 97 to $100 \%$ specificity compared with culture and clinical diagnosis $(14,15,20,22,23)$. These antigen detection systems can be quantitated and therefore used prognostically and to monitor therapy $(1,6,9-11,24)$.

In this study, we compared the CALAS polyclonal latex agglutination kit (Meridian Diagnostics, Cincinnati, Ohio), our standard method for detection of cryptococcal antigen in serum and CSF, with CRYPTO-LEX (Trinity Laboratories, Inc., Raleigh, N.C.), a new monoclonal antibody-based latex agglutination assay. We tested $253 \mathrm{CSF}$ and 327 serum specimens, primarily from HIV-infected patients suspected of having systemic cryptococcal disease, for the presence of cryptococcal antigen. We stored $190 \mathrm{CSF}$ and 232 serum specimens at $4^{\circ} \mathrm{C}$ and tested them within $24 \mathrm{~h}$ of receipt with both CALAS and CRYPTO-LEX. Retrospective analysis was performed with CRYPTO-LEX on $63 \mathrm{CSF}$ and 95 serum specimens stored at $-20^{\circ} \mathrm{C}$. These specimens had been tested previously with CALAS, and recorded results were used for comparison with CRYPTO-LEX results.

The CALAS latex agglutination kit (Meridian Diagnostics) was used in accordance with the instructions provided in the package insert. Prior to testing, all serum samples were treated with pronase for $15 \mathrm{~min}$ at $56^{\circ} \mathrm{C}$ and then boiled for $5 \mathrm{~min}$. CSF specimens were boiled for $5 \mathrm{~min}$ before testing. Specimens demonstrating a $2+$ or greater agglutination reaction were 
TABLE 1. Comparison of CRYPTO-LEX with CALAS for detection of cryptococcal antigen in serum and CSF

\begin{tabular}{crrrrrr}
\hline \multirow{2}{*}{ CRYPTO-LEX result } & \multicolumn{3}{c}{ No. of CALAS-positive specimens } & & \multicolumn{2}{c}{ No. of CALAS-negative specimens } \\
\cline { 2 - 7 } & CSF & Serum & Total & & CSF & Serum \\
\hline Positive & 23 & 44 & 67 & 0 & 0 & Total \\
Negative & 1 & 1 & 2 & 229 & 282 & 511 \\
\hline
\end{tabular}

considered positive, and titers were then determined. The titer was defined as the highest dilution which yielded a $2+$ reaction. The CRYPTO-LEX latex agglutination assay (Trinity Laboratories) utilizes a mouse immunoglobulin M (IgM) monoclonal antibody with reactivity to a repeating epitope in the capsular polysaccharide of $C$. neoformans. As stated by the manufacturer, the reagent reacts with all four serogroups of $C$. neoformans but not with rheumatoid factor since an IgM antibody is used for detection (8). CRYPTO-LEX has been demonstrated to cross-react with antigen from Trichosporon beigelii, as do other cryptococcal antigen latex agglutination tests (8). For CRYPTO-LEX, all CSF specimens were boiled for 5 min. A $300-\mu l$ volume of each serum specimen was treated with an equal volume of protease reagent supplied by the manufacturer and then boiled for $10 \mathrm{~min}$. Fifty microliters of the treated sample (serum or CSF) was placed within a circle on a black disposable card supplied with the kit. One drop of latex sensitized with anti-cryptococcal IgM monoclonal antibody was added to each sample. A control latex suspension was not necessary in this assay. The latex-specimen mixture was rotated for $5 \mathrm{~min}$ at 100 to $110 \mathrm{rpm}$ and read immediately. The titers of all positive specimens $(1+$ or greater) were determined in the same manner as those of the CALASpositive specimens. Regression analysis and determination of correlation coefficients for comparison of titers obtained with the CALAS and CRYPTO-LEX latex tests were done by using the LABSTAT statistical program (Prism Associates, Atlanta, Ga.).

Of 580 specimens, 67 (44 serum and 23 CSF) tested positive and 511 ( 282 serum and $229 \mathrm{CSF}$ ) tested negative with both latex assays (Table 1), resulting in a $99.6 \%$ correlation between the two tests. Two discrepant specimens (one serum and one CSF) were CALAS positive and CRYPTO-LEX negative. Both of these specimens had a titer of $1: 2$ and were from patients with negative cryptococcal cultures. The sensitivity and specificity of CRYPTO-LEX for all specimens (serum and CSF) were 97 and $100 \%$, respectively, with a positive predictive value of $100 \%$ and a negative predictive value of $99.6 \%$. There was no significant difference in these values for either serum or CSF or between refrigerated and frozen specimens. There was no significant difference between CRYPTO-LEX titers obtained with fresh or frozen specimens and CALAS titers. The coefficient of correlation between titers determined with the two assays was poor for serum $(r=0.253)$. However, when two highly discordant serum specimens were removed from the regression analysis, the correlation coefficient became 0.884 . Serum titers that differed by greater than twofold were obtained with 11 of 44 specimens. The CALAS titers were higher in five specimens, and the CRYPTO-LEX titers were higher in six specimens. The coefficient of correlation between the two assays for titers of cryptococcal antigen in CSF was 0.886 . Of 23 CSF specimens, 22 exhibited titers which were within 1 dilution of each other.

Detection of cryptococcal antigen in serum and CSF has become a routine procedure for the diagnosis of cryptococcosis in HIV-infected patients with meningitis or fever of unknown origin. In this study, we compared CRYPTO-LEX, a newly developed latex agglutination test, with CALAS for detection of $C$. neoformans capsular polysaccharide in serum and CSF. CRYPTO-LEX utilizes latex particles sensitized with an IgM monoclonal antibody. Multivalent binding between the IgM antibody and the repeating epitope of the cryptococcal polysaccharide provides increased stability of the antigen-antibody complex compared with polyclonal or monoclonal IgG detection systems. Also, use of an IgM antibody effectively eliminates false-positive reactions with rheumatoid factor, thereby obviating the need for inclusion of a control latex in the assay. No CRYPTO-LEX-positive, CALAS-negative reactions were noted in this study; however, CRYPTO-LEX did not detect two CALAS-positive specimens. The discrepant specimens (one serum and one CSF) had titers of 1:2 and were from patients with negative cryptococcal cultures. The discrepant serum specimen was from a patient who had a cryptococcal antigen titer in serum of 1:801 year prior to the initiation of this study. The patient was receiving therapy and was asymptomatic at the time of collection of the study specimen. This specimen likely represented a false-negative CRYPTO-LEX reaction. The discrepant CSF specimen was from a patient with a positive CSF Venereal Disease Research Laboratory result who was receiving treatment for secondary syphilis. This patient had no previous specimens that were positive for $C$. neoformans, and a CSF specimen submitted 5 months later was negative for cryptococcal antigen. The initial CALAS-positive CSF specimen was considered a false-positive result by the physician. Such false-positive reactions with CALAS may be due to the clarity of the agglutination reaction. It may be difficult to determine the $2+$ agglutination cutoff for a positive CALAS result, and therefore, a $1+$ reaction may be mistakenly read as positive. The fact that any visible agglutination is considered positive with CRYPTO-LEX eliminates the subjective determination of a $2+$ threshold value for positivity. The technologists who routinely read both the CALAS and CRYPTO-LEX reactions found the CRYPTO-LEX results to be much easier to interpret.

The CSF antigen titers obtained with CALAS and CRYPTO-LEX were within 1 dilution in 96\% (22 of 23) of the specimens. The titers differed by more than twofold in 11 (25\%) of 44 serum specimens. CRYPTO-LEX did not produce characteristically higher or lower titers than CALAS. These two tests cannot be used interchangeably for determination of antigen titers, which is consistent with the results obtained in previous comparisons of other latex agglutination assays (14, $15,18,20)$.

Two additional monoclonal antibody-based cryptococcal antigen detection systems are commercially available. These tests have an overall correlation of approximately $98 \%$ compared with CALAS $(14,15,20)$. Advantages and disadvantages of these systems are mentioned here to provide a basis for comparison with the new CRYPTO-LEX system. Pastorex (Sanofi-Diagnostics Pasteur, Marnes-la-Coquette, France), a latex agglutination assay, utilizes an IgG monoclonal antibody for detection of cryptococcal polysaccharide (20). This test 
compared favorably with CALAS. However, since it also uses IgG-based detection, it still necessitates the use of a control latex reagent. The PREMIER (Meridian Diagnostics) enzyme immunoassay for detection of cryptococcal polysaccharide employs a polyclonal antibody capture system with monoclonal detection $(14,15)$. The enzyme immunoassay does not require enzymatic pretreatment of specimens, and it does not react with rheumatoid factor. In comparisons with CALAS, it offered the advantage of somewhat increased sensitivity but false-positive enzyme immunoassay results were also noted $(14,15)$.

Overall, the results obtained with CRYPTO-LEX correlated well $(99.6 \%)$ with those obtained with CALAS. The two tests were similar in terms of specimen preparation and reaction time. We conclude that CRYPTO-LEX is a rapid, reliable, and cost-effective alternative assay for detection of cryptococcal antigen in CSF and serum.

We thank Bruce Clinton of Trinity Laboratories, Inc., for kindly providing the CRYPTO-LEX kits used in this study.

\section{REFERENCES}

1. Bennett, J. E., W. E. Dismukes, R. J. Duma, G. Medoff, M. A. Sande, H. Gallis, J. Leonard, B. T. Fields, M. Bradshaw, H. Haywood, Z. A. McGee, T. R. Cate, C. G. Cobbs, J. F. Warner, and D. W. Alling. 1979. A comparison of amphotericin B alone and combined with flucytosine in the treatment of cryptococcal meningitis. N. Engl. J. Med. 301:126-131.

2. Bennett, J. E., H. F. Hasenclever, and B. S. Tynes. 1964. Detection of cryptococcal polysaccharide in serum and spinal fluid: value in diagnosis and prognosis. Trans. Assoc. Am. Physicians 77:145-150.

3. Bindschadler, D. D., and J. E. Bennett. 1968. Serology of human cryptococcosis. Ann. Intern. Med. 69:45-52.

4. Bloomfield, N., M. A. Gordon, and D. F. Elmendorf. 1963. Detection of Cryptococcus neoformans antigen in body fluids by latex particle agglutination. Proc. Soc. Exp. Biol. Med. 114:64-67.

5. Butler, W. T., D. W. Alling, A. Spickard, and J. P. Utz. 1964. Diagnostic and prognostic value of clinical and laboratory findings in cryptococcal meningitis: a follow-up study of forty patients. $\mathbf{N}$. Engl. J. Med. 270:59-67.

6. Chuck, S. L., and M. A. Sande. 1989. Infections with Cryptococcus neoformans in the acquired immunodeficiency syndrome. N. Engl. J. Med. 321:794-799.

7. Clark, R. A., D. Greer, W. Atkinson, G. T. Valainis, and N. Hyslop. 1990. Spectrum of Cryptococcus neoformans infection in 68 patients infected with human immunodeficiency virus. Rev. Infect. Dis. 12:768-777.

8. Clinton, B. A. (Trinity Laboratories, Inc.). 1994. Personal communication.
9. Dewitt, C. N., P. A. Dickson, and G. W. Holt. 1982. Cryptococcal meningitis. A review of 32 years experience. J. Neurol. Sci. 53:283-292.

10. Diamond, R. D., and J. E. Bennett. 1974. Prognostic factors in cryptococcal meningitis: a study in 111 cases. Ann. Intern. Med. 80:176-181.

11. Dismukes, W. E. 1988. Cryptococcal meningitis in patients with AIDS. J. Infect. Dis. 157:624-628.

12. Eng, R. H. K., E. Bishburg, and S. M. Smith. 1986. Cryptococcal infections in patients with acquired immune deficiency syndrome. Am. J. Med. 81:19-23.

13. Ennis, D. M., and M. S. Saag. 1993. Cryptococcal meningitis in AIDS. Hosp. Pract. 100:99-112.

14. Frank, U. K., S. L. Nishimura, N. C. Li, K. Sugai, D. M. Yajko, W. K. Hadley, and V. L. Ng. 1993. Evaluation of an enzyme immunoassay for detection of cryptococcal capsular polysaccharide antigen in serum and cerebrospinal fluid. J. Clin. Microbiol. 31:97-101.

15. Gade, W., S. W. Hinnefeld, L. S. Babcock, P. Gilligan, W. Kelly, K. Wait, D. Greer, M. Pinilla, and R. L. Kaplan. 1991. Comparison of the PREMIER cryptococcal antigen enzyme immunoassay and the latex agglutination assay for detection of cryptococcal antigens. J. Clin. Microbiol. 29:1616-1619.

16. Goodman, J. S., L. Kaufman, and M. G. Koenig. 1971. Diagnosis of cryptococcal meningitis: value of immunologic detection of cryptococcal antigen. N. Eng. J. Med. 285:434-436.

17. Gordon, M. A., and D. K. Vedder. 1966. Serologic tests in diagnosis and prognosis of cryptococcosis. JAMA 197:961-967.

18. Hamilton, J. R., A. Noble, D. W. Denning, and D. A. Stevens. 1991. Performance of cryptococcus antigen latex agglutination kits on serum and cerebrospinal fluid specimens of AIDS patients before and after pronase treatment. J. Clin. Microbiol. 29:333-339.

19. Kaufman, L., and S. Blumer. 1968. Value and interpretation of serologic tests for the diagnosis of cryptococcosis. Appl. Microbiol. 16:1907-1912.

20. Temstet, A., P. Roux, J.-L. Poirot, O. Ronin, and F. Dromer. 1992. Evaluation of a monoclonal antibody-based latex agglutination test for diagnosis of cryptococcosis: comparison with two tests using polyclonal antibodies. J. Clin. Microbiol. 30:2544-2550.

21. Walter, J. E., and R. D. Jones. 1968. Serodiagnosis of clinical cryptococcosis. Am. Rev. Respir. Dis. 97:275-282.

22. Warren, R. J., A. Perceval, and B. W. Dwyer. 1993. Comparative evaluation of cryptococcal latex tests. Pathology 25:76-80.

23. Wu, T. C., and S. Y. Koo. 1983. Comparison of three commercial cryptococcal latex kits for detection of cryptococcal antigen. J. Clin. Microbiol. 18:1127-1130.

24. Zuger, A., E. Louie, R. S. Holzman, M. S. Simberkoff, and J. J. Rahal. 1986. Cryptococcal disease in patients with the acquired immunodeficiency syndrome. Diagnostic features and outcome of treatment. Ann. Intern. Med. 104:234-240. 